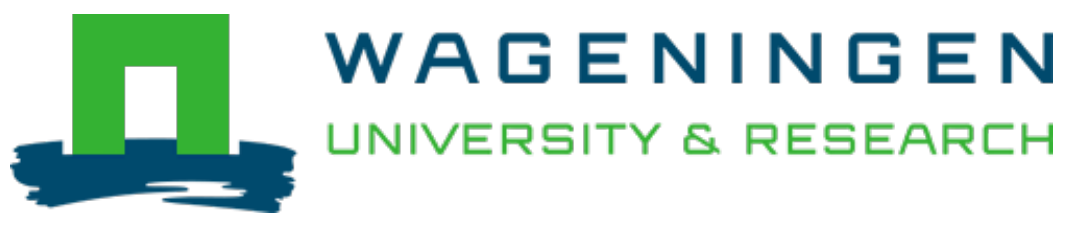

\title{
Cardiorespiratory fitness, muscular fitness and mental health in older adolescents: A multi-level cross-sectional analysis
}

\author{
Preventive Medicine \\ Janssen, Amy; Leahy, Angus A.; Diallo, Thierno M.O.; Smith, Jordan J.; Kennedy, Sarah G. et al \\ https://doi.org/10.1016/j.ypmed.2020.105985
}

This publication is made publicly available in the institutional repository of Wageningen University and Research, under the terms of article $25 \mathrm{fa}$ of the Dutch Copyright Act, also known as the Amendment Taverne. This has been done with explicit consent by the author.

Article 25 fa states that the author of a short scientific work funded either wholly or partially by Dutch public funds is entitled to make that work publicly available for no consideration following a reasonable period of time after the work was first published, provided that clear reference is made to the source of the first publication of the work.

This publication is distributed under The Association of Universities in the Netherlands (VSNU) 'Article $25 \mathrm{fa}$ implementation' project. In this project research outputs of researchers employed by Dutch Universities that comply with the legal requirements of Article $25 \mathrm{fa}$ of the Dutch Copyright Act are distributed online and free of cost or other barriers in institutional repositories. Research outputs are distributed six months after their first online publication in the original published version and with proper attribution to the source of the original publication.

You are permitted to download and use the publication for personal purposes. All rights remain with the author(s) and / or copyright owner(s) of this work. Any use of the publication or parts of it other than authorised under article $25 \mathrm{fa}$ of the Dutch Copyright act is prohibited. Wageningen University \& Research and the author(s) of this publication shall not be held responsible or liable for any damages resulting from your (re)use of this publication.

For questions regarding the public availability of this publication please contact openscience.library@wur.nl 


\title{
Cardiorespiratory fitness, muscular fitness and mental health in older adolescents: A multi-level cross-sectional analysis
}

\author{
Amy Janssen $^{\mathrm{a}}$, Angus A. Leahy ${ }^{\mathrm{b}}$, Thierno M.O. Diallo ${ }^{\mathrm{c}}$, Jordan J. Smith ${ }^{\mathrm{b}}$, Sarah G. Kennedy ${ }^{\mathrm{b}}$, \\ Narelle Eather $^{\mathrm{b}}$, Myrto F. Mavilidi ${ }^{\mathrm{b}}$, Annemarie Wagemakers ${ }^{\mathrm{a}}$, Mark J. Babic ${ }^{\mathrm{b}}$, David R. Lubans ${ }^{\mathrm{b}, *}$ \\ ${ }^{a}$ Group Health and Society, Department of Social Sciences, Wageningen University \& Research, the Netherlands \\ ${ }^{\mathrm{b}}$ Priority Research Centre in Physical Activity and Nutrition, School of Education, University of Newcastle, Callaghan, New South Wales, Australia \\ ${ }^{\mathrm{c}}$ School of Social Sciences and Psychology, Western Sydney University, Penrith, NSW, Australia
}

\section{A R T I C L E IN F O}

\section{Keywords:}

Muscular fitness

Cardiorespiratory fitness

Well-being

Internalizing problems

Adolescents

\begin{abstract}
A B S T R A C T
Physical activity interventions that promote cardiorespiratory (CRF) and muscular fitness (MF) may improve mental health in young adolescents. However, less is known about the links between fitness and mental health in older adolescents, as they are an understudied population. In addition, the association between MF and adolescents' mental health is less clear than it is for CRF. Our primary aim was to investigate whether MF is independently associated with mental health in a sample of older adolescents. Our secondary aim was to determine if the association between fitness and mental health was moderated by sex, socio-economic status (SES) or weight status. Participants were 670 students (16.0 [0.4] years, 44.6\% female) from 20 secondary schools in New South Wales, Australia. Mental health (well-being and internalizing problems) was self-reported using the Short Warwick-Edinburgh Mental Wellbeing Scale and the Strengths and Difficulties Questionnaire. We assessed CRF using the Progressive Aerobic Cardiovascular Endurance Run and MF using the push-up and standing long jump tests. After controlling for CRF, MF was not associated with mental health. CRF was associated with wellbeing $(\beta=0.20, p<.001)$ and internalizing problems $(\beta=-0.27, p<.001)$. The strength of association between CRF and mental health was stronger in girls, than boys. No interaction effects were observed for SES or weight status. Although cross-sectional, our findings provide further evidence of the potential benefits of CRF for adolescents' mental health (i.e., well-being and internalizing problems), particularly girls. However, MF may be less relevant for mental health among this population.
\end{abstract}

\section{Introduction}

Mental health is defined as 'a state in which the individual realizes his or her own abilities, can cope with the normal stresses of life, can work productively and fruitfully, and is able to make a contribution to his or her community' (World Health Organisation, 2005). Importantly, indicators of mental health can include both positive (i.e., well-being) and negative (i.e., ill-being) constructs. Common indicators of wellbeing include self-esteem, positive affect, life satisfaction, quality of life and psychological resilience. Alternatively, ill-being constructs include preclinical psychological states (e.g., high levels of perceived stress) and clinically diagnosed mental disorders (e.g., depression and anxiety) (Lubans et al., 2016a).

Mental disorders place a significant burden on individuals and society (Whiteford et al., 2013). Evidence suggests secular increases in stress and anxiety among adolescents in recent decades (Bor et al., 2014), with 'school-related stress' identified as a major driver of this change (Sweeting et al., 2010). Adolescent girls typically report experiencing more stressful events and higher levels of depression and anxiety, compared to boys (Rudolph, 2002). The period of late adolescence (15-18 years) is one in which young people may be particularly susceptible to mental health disorders, with the normal challenges of maturation (i.e., changing social roles, formation of identity, commencement of paid work, and physical changes) compounded by the pressure to perform in final school examinations (Beauchamp et al., 2018). These changes are occurring against a backdrop of decreases in physical activity, such as the removal of mandatory physical education for senior school students (Beauchamp et al., 2018).

Participation in physical activity can enhance adolescents' wellbeing and reduce indicators of internalizing problems (Biddle et al.,

\footnotetext{
* Corresponding author at: Priority Research Centre for Physical Activity and Nutrition, School of Education, Faculty of Education and Arts, University of Newcastle, Callaghan, NSW 2308, Australia.

E-mail address: David.Lubans@newcastle.edu.au (D.R. Lubans).
} 
2019). As suggested by Lubans et al. (2016a), the time (i.e., how long), type (i.e., what), intensity (i.e., how hard) and context (i.e., where and with whom) of physical activity may moderate the effects on mental health outcomes, which are thought to operate via a number of possible neurobiological, psychosocial and behavioral mechanisms. For example, in a large sample of adolescents, vigorous intensity physical activity was more strongly associated with mental health benefits than activity of light and moderate intensity (Costigan et al., 2019). Of note, participating in physical activity of sufficient intensity to improve health-related fitness (i.e., cardiorespiratory fitness [CRF], muscular fitness [MF] and body composition) may be necessary to induce neurobiological adaptations. According to the cross-stressor-adaptation (CSA) hypothesis (Kjær, 1992), exposure to a physical stress such as vigorous exercise, triggers a response similar to that following exposure to a psychosocial stressor (i.e., increase in heart rate, respiration, blood cortisol). The CSA hypothesis posits that the beneficial adaptation of the hypothalamic-pituitary-adrenocortical axis and the sympathoadrenal medullary system during physical exercise can generalize to psychosocial stressors and may improve mental health (Mücke et al., 2018).

Although higher levels of CRF have been positively associated with indicators of well-being (Eddolls et al., 2018), and negatively associated with internalizing problems (Rieck et al., 2013; Ruggero et al., 2015), findings have been inconsistent and most of the research has focused on children and young adolescents (i.e., 12 to 15 years) (Lang et al., 2018a). For example, some studies have found positive associations between CRF and mental health (Kelly et al., 2011), while others have reported non-significant or even negative associations (Richards et al., 2014; Ransdell et al., 2004). It has also been suggested that MF may have unique mental health benefits for adolescents (Biddle et al., 2019; Smith et al., 2014; Yeatts et al., 2017). A recent study found that absolute upper- and lower-body muscular strength was positively associated with self-esteem in a small sample of overweight/obese children (Rodriguez-Ayllon et al., 2018). In another study involving adolescents, MF was found to be positively associated with physical self-perceptions (i.e., perceived appearance and sport competence) (Lubans and Cliff, 2011), which are known to generalize to global self-esteem. However, previous studies have failed to control for CRF levels in their statistical analyses, preventing us from drawing conclusions regarding the unique benefits of MF for adolescents' mental health. Additional limitations include the failure to measure both positive and negative indicators of mental health and the focus on children and young adolescents.

Examining the associations between fitness and mental health in older adolescents is particularly important as this is when physical activity levels decline dramatically (Dumith et al., 2011) and many mental health disorders emerge (Kessler et al., 2007). Therefore, the primary aim of our study was to investigate whether MF is independently associated with mental health (i.e., well-being and internalizing problems) in older adolescents. Our secondary aim was to determine if associations between fitness and mental health were moderated by sex, socio-economic status (SES) and weight status.

\section{Methods}

\subsection{Design and participants}

Baseline data from the Burn 2 Learn cluster randomized controlled trial were used for this study (Leahy et al., 2019). The study sample included data from 670 adolescents in Grade 11 (mean age $=16.0$ [0.4] years) from 20 secondary schools participating in a two-arm parallel group cluster randomized controlled trial. Burn 2 Learn is a school-based physical activity intervention designed for Grade 11 students from secondary schools in New South Wales (NSW), Australia. The study is registered with the Australian New Zealand Clinical Trials Registry (ACTRN12617000544370). Ethics approval was provided by the University of Newcastle (H-2016-0424) and the NSW Department of
Education (SERAP: 2017116) human research ethics committees.

New South Wales (NSW) government secondary schools with senior school students (i.e., Grades 11 and 12, students aged 16-18) from four geographical areas (i.e., Hunter-Central Coast, Northern and Western Sydney, and New England) were eligible to participate in the study. Schools from the selected geographical regions are broadly representative of urban and regional schools in NSW. Two Grade 11 teachers from each school were recruited and asked to invite their students to participate in the study (there were no restrictions regarding the teaching discipline). The consent rate for students who agreed to participate in the study was $79.4 \%$. Full details of the study protocols and pilot findings are reported elsewhere (Leahy et al., 2019; Leahy et al., 2019b).

\subsection{Measures}

\subsubsection{Data collection}

Baseline data were collected in schools by trained research assistants from February to April in 2018 and 2019, respectively. Measures of self-report (well-being and internalizing problems, and demographic information) were administered using iPads under exam-like conditions. Fitness assessments were conducted in the schools' gym in a sensitive manner by same-sex research assistants.

\subsubsection{Well-being}

The Short Warwick-Edinburgh Mental Wellbeing Scale was used to assess psychological well-being (Clarke et al., 2011) and consisted of seven statements relating to indicators of well-being (i.e., global selfesteem, psychological and subjective well-being, quality of life and psychological resilience). Statements are scored on a five-point scale ranging from 1 'None of the time' to 5 'All of the time', and are summed to produce a well-being composite.

\subsubsection{Internalizing problems}

The 'emotional' and 'peer problems' subscales (both include five items) from the Strengths and Difficulties Questionnaire (SDQ) were combined to provide a measure of internalizing problems (Goodman, 1997), with items scored on a three-point scale (i.e., $0=$ 'Not true', $1=$ 'Somewhat true', and 2 = 'Certainly true').

\subsubsection{Cardiorespiratory fitness}

FITNESSGRAM $^{\circledR}$ testing procedures were used (Cooper Institute for Aerobics Research, 2004) to assess CRF using the Progressive Aerobic Cardiovascular Endurance Run (PACER). The PACER is the most widely accepted field-based measure of CRF and demonstrates high levels of reliability and validity (Lang et al., 2018b).

\subsubsection{Muscular fitness}

The 90-degree push-up test was used to assess upper body muscular endurance, which has acceptable validity and reliability (Lubans et al., 2011). Lower body muscular power was assessed using the standing long jump test (Castro-Pinero et al., 2010). A MF index was calculated using the results from the two MF tests (i.e., distance jumped and number of successful repetitions). Scores for the individual tests were standardized [(value - mean)/standard deviation] and then summed to create the MF index.

\subsubsection{Body-mass index}

Body mass index (BMI) was calculated using the standard equation (weight $[\mathrm{kg}] /$ height $[\mathrm{m}]^{2}$ ). BMIz-scores were calculated using the lambdamu-sigma method (International Obesity Task Force cut-offs) to determine weight status (Cole and Lobstein, 2012). Weight and height were assessed using standardized protocols.

\subsubsection{Demographics}

Sex [i.e., female, male or other], area-level SES (residential postal 
code), place of birth, language spoken at home, cultural background, and Indigenous status were collected via questionnaires. SES was determined using the Index of Relative Socioeconomic Disadvantage from the Australian Bureau of Statistics census-based Socio-Economic Indexes for Areas (Australian Bureau of Statistics, 2001). The SEIFA index (scale $1=$ lowest to $10=$ highest) summarizes the characteristics of people and households within an area. For descriptive purposes, area-level SEIFA values were organized into tertiles (low $=1$ to 3 , medium $=4$ to 7 and high 8 to 10 ).

\subsection{Power calculation}

The original study power calculation was conducted to determine the sample size needed to detect a change in the primary outcome CRF. Baseline values from our pilot trial (Leahy et al., 2019b) and conservative intraclass correlation coefficient (ICC) values of 0.20 and 0.03 were used to account for clustering at the class- and school-levels, respectively (Heo and Leon, 2008). Assuming $80 \%$ power at a $5 \%$ significance level we estimated that 560 students (i.e., 2 classes of 14 students from each of 20 schools) would provide adequate statistical power. Posteriori power estimates were computed using simulatedbased method along with Wald test in Mplus. The resulting power estimates for the regression model predicting well-being ranged from 0.14 for MF to 0.93 for CRF. The power estimates for the regression model predicting internalizing problems ranged from 0.10 for MF to 0.95 for CRF.

\subsection{Statistical analyses}

Analyses were performed using the Mplus 8.3 program (Muthén and Muthén, 1998-2017). Means and standard deviations were calculated for all normally distributed variables. Bivariate correlations were used to examine correlations between health-related fitness and mental health outcomes (alpha levels set at $p<.05$ ). The robust maximum likelihood estimation procedure was used to account for missing data and the non-independence of students nested within schools by adjusting the standard errors using a sandwich estimator. Linear regression models were run separately for the dependent variables well-being and internalizing problems. All models included the independent variables CRF and MF and the following covariates: sex, SES and BMI. Moderation effects were investigated by testing for the interaction effect of sex, SES and weight status on the association between CRF and mental health outcomes. If the interaction term in the regression model was statistically significant $(p<.10)$, we ran separate regression models for the sub-groups (e.g., boys and girls) and report the standardized coefficients. Finally, intraclass correlation coefficients (ICC) were calculated for key variables of interest (i.e., CRF, MF, well-being and internalizing problems).

\section{Results}

\subsection{Descriptive statistics}

Participants' characteristics are reported in Table 1. ICC values for CRF (ICC $=0.12$ ) and MF (ICC $=0.12$ ) were larger than those observed for well-being (ICC $=0.04$ ) and internalizing problems $(\mathrm{ICC}=0.04)$

\subsection{Bivariate correlations between variables}

As illustrated in Table 2, CRF was significantly associated with MF, well-being and internalizing problems. The strength of the correlation between CRF and MF was large $(r=0.72, p<.001)$, while the strength of the association between CRF and well-being $(r=0.27$, $p<.001)$ and internalizing problems $(r=-0.33, p<.001)$ was small to moderate. BMI was not significantly associated with mental
Table 1

Characteristics of study sample.

\begin{tabular}{|c|c|}
\hline Characteristics & $\begin{array}{l}\text { Total } \\
(n=670)\end{array}$ \\
\hline Age, mean (SD), $\mathrm{y}^{\mathrm{a}}$ & $16.0(0.4)$ \\
\hline Female participants, $\mathrm{n}(\%)^{\mathrm{a}}$ & $299(44.6)$ \\
\hline Born in Australia, $\mathrm{n}(\%)^{\mathrm{a}}$ & $587(88.1)$ \\
\hline English spoken at home, $\mathrm{n}(\%)^{\mathrm{a}}$ & $618(92.8)$ \\
\hline \multicolumn{2}{|l|}{ Cultural background, $\mathrm{n}(\%)^{\mathrm{a}}$} \\
\hline Australian & $469(70.4)$ \\
\hline European & $67(10.1)$ \\
\hline African & $6(0.9)$ \\
\hline Asian & $39(5.9)$ \\
\hline Middle Eastern & $7(1.1)$ \\
\hline Other & $78(11.7)$ \\
\hline Indigenous decent, $\mathrm{n}(\%)^{\mathrm{a}}$ & $61(9.2)$ \\
\hline \multicolumn{2}{|c|}{ Socioeconomic status based on household postcode, $\mathrm{n}(\%)^{\mathrm{b}}$} \\
\hline Low & $129(19.4)$ \\
\hline Medium & $339(51.1)$ \\
\hline High & $196(29.5)$ \\
\hline \multicolumn{2}{|l|}{ Weight status, n (\%) ${ }^{\mathrm{c}}$} \\
\hline Underweight & $26(3.9)$ \\
\hline Healthy weight & $445(67.3)$ \\
\hline Overweight & $134(20.3)$ \\
\hline Obese & $56(8.5)$ \\
\hline
\end{tabular}

${ }^{\text {a }}$ Four students did not answer the demographic questions.

b Socioeconomic status determined by population tertile using socio-economic indexes for areas of relative socioeconomic disadvantage based on residential postcode; six participants did not provide their residential postcode.

c Nine participants were not measured for weight status.

health.

\subsection{Regression models predicting well-being}

The multi-level regression models predicting well-being explained $8 \%$ of the variance (Table 3$)$. CRF $(\beta=0.201, p<.001)$ was significantly associated with well-being. After adjustment for age, sex, CRF and MF, BMI z-score $(\beta=0.063, p=.022)$ was also positively associated with well-being, suggesting that adolescents with higher BMI reported higher levels of well-being. The sex interaction term was statistically significant for the CRF $(\mathrm{B}=0.059, p<.001)$, but not for MF $(\mathrm{B}=0.863, p=.056)$. Separate regression models demonstrated that CRF was more strongly associated with well-being in girls $(\beta=0.257$, $p<.001)$ than boys $(\beta=0.112, p=.017)$. SES did not moderate the cross-sectional association between MF $(\mathrm{B}=0.001, p=.783)$ or $\mathrm{CRF}$ ( $\mathrm{B}=0.001, p=.610$ ) and well-being. Similarly, the weight status interaction term was not significant for MF $(\mathrm{B}=0.001, p=.378)$ or $\mathrm{CRF}(\mathrm{B}=0.001, p=.386)$.

\subsection{Regression models predicting internalizing problems}

The multi-level regression models predicting internalizing problems explained $11.5 \%$ of the variance. CRF $(\beta=-0.204, p<.001)$ and sex ( $\beta=0.116, p=.014$ ) were significantly associated with internalizing problems (Table 4$)$. The sex interaction term was statistically significant for CRF ( $\mathrm{B}=-0.041, p<.001$ ), but not for MF ( $=-.458$, $\mathrm{p}$ $=.086)$ Separate regression models demonstrated that $\mathrm{CRF}$ was more strongly associated with internalizing problems in girls $(\beta=-0.276$, $p<.001)$ than boys $(\beta=-0.105, p=.038)$. The SES interaction term was not significant for MF ( $=-0.001, p=.631$ ) or $\mathrm{CRF}$ ( $\mathrm{B}=0.001, p=.828)$. Similarly, weight status did not moderate the cross-sectional association between $\mathrm{MF}(\mathrm{B}=-0.001, p=.227)$ or $\mathrm{CRF}$ $(\mathrm{B}=0.001, p=.136)$ and internalizing problems.

\section{Discussion}

The primary aim of our study was to determine if MF was associated 
Table 2

Descriptive statistics and bivariate correlations between variables.

\begin{tabular}{|c|c|c|c|c|c|c|c|c|}
\hline \multirow[t]{2}{*}{ Variable } & \multicolumn{3}{|c|}{ Descriptive statistics } & \multicolumn{5}{|c|}{ Bivariate correlations, $r$ ( $p$-values) } \\
\hline & $n$ & M & SD & 1 & 2 & 3 & 4 & 5 \\
\hline 1. Well-being & 666 & 24.4 & 4.91 & - & & & & \\
\hline 2. Internalizing problems & 666 & 5.5 & 3.2 & $\begin{array}{l}-0.49 \\
(<0.001)\end{array}$ & - & & & \\
\hline 3. BMI z-score & 661 & 0.71 & 1.03 & $\begin{array}{l}-0.01 \\
(0.717)\end{array}$ & $\begin{array}{l}-0.01 \\
(0.763)\end{array}$ & - & & \\
\hline 4. Cardiorespiratory fitness & 605 & 48.8 & 25.4 & $\begin{array}{l}0.27 \\
(<0.001)\end{array}$ & $\begin{array}{l}-0.33 \\
(<0.001)\end{array}$ & $\begin{array}{l}-0.32 \\
(<0.001)\end{array}$ & - & \\
\hline 5. Muscular fitness & 620 & 0.02 & 1.83 & $\begin{array}{l}0.24 \\
(<0.001)\end{array}$ & $\begin{array}{l}-0.30 \\
(<0.001)\end{array}$ & $\begin{array}{l}-0.19 \\
(<0.001)\end{array}$ & $\begin{array}{l}0.72 \\
(<0.001)\end{array}$ & - \\
\hline
\end{tabular}

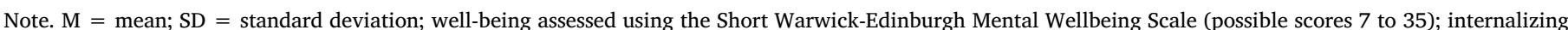

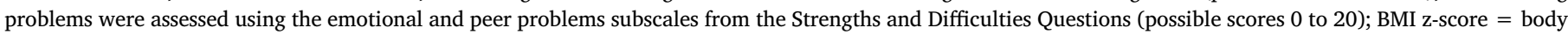

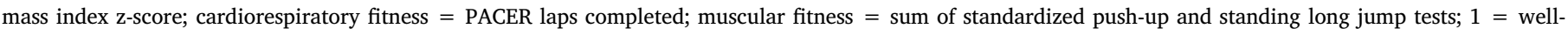
being, 2 = internalizing problems, 3 = BMI z-score, $4=$ cardiorespiratory fitness, $5=$ muscular fitness.

Table 3

Regression model predicting adolescent well-being.

\begin{tabular}{|c|c|c|c|c|}
\hline Variables & Unstandardized estimate B & Standard error of estimate B & Standardized $\beta$ & $p$ Value \\
\hline Age & 0.468 & 0.507 & 0.044 & 0.356 \\
\hline Sex & -0.352 & 0.628 & -0.037 & 0.575 \\
\hline Socio-economic status & -0.011 & 0.007 & -0.057 & 0.116 \\
\hline BMI z-score & 0.288 & 0.125 & 0.063 & 0.021 \\
\hline Cardiorespiratory fitness & 0.038 & 0.007 & 0.201 & $<0.001$ \\
\hline Muscular fitness & 0.152 & 0.166 & 0.058 & 0.360 \\
\hline R square & & & & 0.080 \\
\hline
\end{tabular}

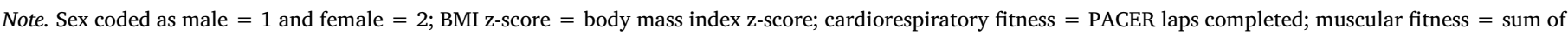
standardized push-up and standing long jump tests; $B=$ unstandardized coefficient and $\beta=$ standardized coefficient.

with mental health (i.e., well-being and internalizing problems) independently of CRF in older adolescents. Although MF was significantly associated with both mental health outcomes in the bivariate correlations, the association was no longer statistically significant after adjusting for sex, SES, BMI and CRF. Our secondary aim was to determine if the association between fitness (both CRF and MF) and mental health was moderated by sex, SES and weight status. Notably, the strength of the association between CRF and mental health was significantly stronger in girls, compared to boys. No interaction effects were observed for SES or weight status.

We did not find an independent association between MF and mental health in our sample of older adolescents. Previous studies examining the associations between MF and mental health in adolescents have produced mixed findings (Rodriguez-Ayllon et al., 2018; Lubans et al., 2016b; Andersen et al., 2017) and none focused on older adolescents in their final years of school. For example, higher muscular strength was associated with more positive mental health outcomes, including lower level of stress and negative affect, as well as higher levels of self-esteem and optimism in a small sample of overweight children (RodriguezAyllon et al., 2018). Alternatively, CRF but not MF was positively associated with all five health-related quality of life domains in Norwegian children (Andersen et al., 2017). Powerful evidence for the benefits of MF for mental health can be observed in a 24-year longitudinal study involving more than one million male adolescents (16-19 years) (Ortega et al., 2012). In this study, muscular strength in adolescence was linked to $20-30 \%$ lower risk of death from suicide, and $15-65 \%$ less chances to be diagnosed with any psychiatric disease as an adult (Ortega et al., 2012). However, it is important to note that this study (Ortega et al., 2012) did not adjust for the potential confounding effect of CRF, and risk reductions for suicide- and substance-abuse related mortality were also found with higher CRF in this cohort (Högström et al., 2015).

Internalizing symptoms often emerge during adolescence and if untreated, may manifest as depression and anxiety. Consistent with previous literature, we observed a moderate negative association between CRF and internalizing problems (Rieck et al., 2013; Ruggero et al., 2015). However, the majority of research has focused on children and young adolescents. For example, Rieck et al. (2013) reported that children with higher levels of CRF reported significantly lower levels of depression. In a longitudinal study concerning middle school students

Table 4

Regression model predicting internalizing problems.

\begin{tabular}{|c|c|c|c|c|}
\hline Variables & Unstandardized estimate B & Standard error of estimate B & Standardized $\beta$ & $p$ Value \\
\hline Age & -0.139 & 0.269 & -0.020 & 0.605 \\
\hline Sex & 0.736 & 0.304 & 0.116 & 0.014 \\
\hline Socio-economic status & -0.009 & 0.005 & -0.074 & 0.055 \\
\hline BMI z-score & -0.030 & 0.109 & -0.010 & 0.783 \\
\hline Cardiorespiratory fitness & -0.026 & 0.005 & -0.204 & $<0.001$ \\
\hline Muscular fitness & -0.088 & 0.094 & -0.050 & 0.354 \\
\hline R square & & & & 0.115 \\
\hline
\end{tabular}

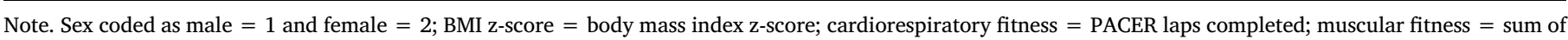
standardized push-up and standing long jump tests; $B=$ unstandardized coefficient and $\beta=$ standardized coefficient. 
(Ruggero et al., 2015), CRF in sixth grade (mean age 11.6 years) was associated with lower levels of depression by seventh grade in girls, but not in boys (although the effects were in the same direction). Another longitudinal study involving middle school students (mean age 11.4 years) revealed that higher levels of physical fitness, including CRF and MF, were associated with improved mental health (i.e., lower levels of depression, peer rejection, loneliness, internalizing symptoms as well as higher levels of adaptive functioning, externalizing symptoms, selfworth, and perceived competence) (LaVigne et al., 2016). To our knowledge, this is the first study to identify an inverse association between CRF and internalizing problems in older adolescents. These findings have important implications for physical activity promotion in this age group.

Sex was a significant moderator of the association between CRF and mental health in our study. This finding may be explained by the interpersonal stresses experienced by adolescent girls as well as their physical activity profiles. Consistent with previous research, girls in our study reported higher levels of psychological distress and displayed lower levels of fitness, than boys (Ruggero et al., 2015; Yeatts et al., 2017). Evidence suggests that girls typically exhibit more negative emotional responses to stressful events, in the form of anxiety and depression, compared to boys (Rudolph, 2002). Overall, girls tend to be more "vulnerable" than boys throughout adolescence and may be more susceptible to the consequences of poor fitness. The majority of crosssectional (Lang et al., 2018a) and experimental (Brown et al., 2013) studies examining the association and impact of CRF and MF has involved younger adolescents and produced modest effect sizes. Additional studies involving older adolescents are needed and such studies may yield larger effects, particularly among girls and those with low levels of fitness and poor mental health.

Consistent with our findings, several studies have demonstrated that CRF is positively associated with various indicators of well-being in young adolescents (Riiser et al., 2014; Morales et al., 2013). For example, Riiser et al. (2014) demonstrated that CRF was significantly associated with health-related quality of life in Norwegian adolescents. Also, CRF in girls and MF in boys, were more strongly associated with health-related quality of life dimensions in Spanish children (Morales et al., 2013). It is perhaps worth noting that the Spanish study measured MF using a handgrip strength and the standing broad jump tests, which measure strength and power, respectively (Morales et al., 2013). In the current study, we used thestanding long jump and push-up tests to determine MF and it is worth noting that other measures might produce different results. We intended to provide a proxy measure of 'overall' MF by creating a composite score encompassing both lower and upper body regions and both maximal strength/power (standing long jump) and muscular endurance (push-ups) domains. However, it remains possible that certain dimensions of MF are more strongly related to mental health than others, and the lack of association in the present study is a measurement issue. As such, future research could evaluate associations between specific MF and mental health in adolescents, using criterion measures (e.g., 1 repetition maximum testing) (Ruiz et al., 2011).

Interestingly, BMI z-scores were positively associated with internalizing problems in the bivariate correlations, but this association was reversed when MF and CRF were included in the regression model. It is possible that high levels of MF and CRF act as a protective barrier against internalizing problems among adolescents with high BMI zscores. Similar findings were demonstrated in a longitudinal study involving adults, where no measure of fatness was associated with the onset of depressive symptoms after controlling for CRF (Becofsky et al., 2015). However, due to the relatively small magnitude of effect in the present study, we cannot discount the possibility of type II error.

\subsection{Strengths and limitations}

The strengths of this study include a unique study population, field- based measures for CRF and MF, multi-level regression models adjusting for clustering of effects at the school level and validated questionnaires for different mental health outcomes. However, some limitations should be acknowledged. First, as this is a cross-sectional study, causation cannot be established. Randomized controlled trials are needed to clarify the independent effects of CRF and MF on mental health in older adolescents. Second, our study measured broad domains of mental health (i.e., well-being and internalizing problems), but did not specify the specific indicators of mental health within those domains (e.g., anxiety and depression). Evidence for the benefits of fitness for the prevention of specific mental health disorders in young people will support health promotion efforts. Third, our posterior power calculation revealed lower statistical power to detect associations for MF compared to CRF. As such, due to the relatively small magnitude of effect in the present study, we cannot discount the possibility of type II error. Finally, although the PACER is considered the most appropriate field-based measure for evaluating CRF, it is difficult to determine whether maximal effort is reached.

\section{Conclusion}

MF was not independently associated with mental health in a sample of older adolescents aged 15-17 years. It is possible that a measure of muscular strength (e.g., handgrip test) would produce a different result, as we found that our MF index was strongly correlated with CRF in the study sample. After adjustment for CRF and MF, BMI was not associated with internalizing problems and positively associated with well-being. Consistent with previous research, CRF was moderately associated with mental health in older adolescents, with stronger associations observed in girls.

\section{CRediT authorship contribution statement}

Amy Janssen:Writing - original draft.Angus Leahy:Data curation, writing - review \& editing.Thierno M.O. Diallo:Formal analysis.Jordan J. Smith:Conceptualization, funding acquisition, methodology.Sarah Kennedy:Data curation, project administration, writing - review \& editing. Narelle Eather:Conceptualization, funding acquisition, methodology.Myrto Mavilidi:Data curation, project administration, , writing - review \& editing.Annemarie Wagemakers:Writing - review \& editing.Mark J. Babic: Writing review \& editing.David R. Lubans: Conceptualization, data curation, funding acquisition, investigation, methodology.

\section{Acknowledgments}

This study was funded by a National Health and Medical Research Council (NHMRC) Project Grant (APP1120518). The authors would like to thank Charles Hillman, Philip Morgan, Ron Plotnikoff, Chris Lonsdale, Michael Nilsson and, the NSW Department of Education, with special thanks to Ross Morrison and James Boyer. DRL is supported by an NHMRC Senior Research Fellowship (APP1154507). Finally, we would like to thank the schools, teachers, parents, and study participants for their involvement.

\section{References}

Andersen, J.R., Natvig, G.K., Aadland, E., et al., 2017. Associations between health-related quality of life, cardiorespiratory fitness, muscle strength, physical activity and waist circumference in 10-year-old children: the ASK study. Qual. Life Res. 26 (12), 3421-3428.

Australian Bureau of Statistics, 2001. Socio-Economic Indexes for Areas: Census of Population and Housing. Australian Bureau of Statistics, Canberra, Australia.

Beauchamp, M.R., Puterman, E., Lubans, D.R., 2018. Physical inactivity and mental health in late adolescence. JAMA Psychiat. 75 (6), 543-544. https://doi.org/10. 1001/jamapsychiatry.2018.0385.

Becofsky, K.M., Sui, X., D-c, L., et al., 2015. A prospective study of fitness, fatness, and depressive symptoms. Am. J. Epidemiol. 181 (5), 311-320. https://doi.org/10.1093/ 
aje/kwu330.

Biddle, S.J., Ciaccioni, S., Thomas, G., et al., 2019. Physical activity and mental health in children and adolescents: an updated review of reviews and an analysis of causality. Psychol. Sport Exerc. 42, 146-155.

Bor, W., Dean, A.J., Najman, J., et al., 2014. Are child and adolescent mental health problems increasing in the 21st century? A systematic review. Aust. N. Z. J. Psychiatry 48 (7), 606-616.

Brown, H.E., Pearson, N., Braithwaite, R.E., et al., 2013. Physical activity interventions and depression in children and adolescents: a systematic review and meta-analysis. Sports Med. 43 (3), 195-206. https://doi.org/10.1007/s40279-012-0015-8.

Castro-Pinero, J., Ortega, F.B., Artero, E.G., et al., 2010. Assessing muscular strength in youth: usefulness of the standing long jump as a general index of muscular fitness. J. Strength Cond. Res. 24, 1810-1817.

Clarke, A., Friede, T., Putz, R., et al., 2011. Warwick-Edinburgh Mental Well-being Scale (WEMWBS): validated for teenage school students in England and Scotland. A mixed methods assessment. BMC Public Health 11 (1), 487.

Cole, T.J., Lobstein, T., 2012. Extended international (IOTF) body mass index cut-offs for thinness, overweight and obesity. Pediatr. Obes. 7 (4), 284-294.

Cooper Institute for Aerobics Research, 2004. The Prudential Fitnessgram: Test Administration Manual, 3rd edition. Human Kinetics, Campaign, IL.

Costigan, S.A., Lubans, D.R., Lonsdale, C., et al., 2019. Associations between physical activity intensity and well-being in adolescents. Prev. Med. 125, 55-61.

Dumith, S.C., Gigante, D.P., Domingues, M.R., et al., 2011. Physical activity change during adolescence: a systematic review and a pooled analysis. Int. J. Epidemiol. 40 (3), 685-698. https://doi.org/10.1093/ije/dyq272.

Eddolls, W.T., McNarry, M.A., Lester, L., et al., 2018. The association between physical activity, fitness and body mass index on mental well-being and quality of life in adolescents. Qual. Life Res. 27 (9), 2313-2320.

Goodman, R., 1997. The strengths and difficulties questionnaire: a research note. J. Child Psychol. Psychiatry 38 (5), 581-586.

Heo, M., Leon, A.C., 2008. Statistical power and sample size requirements for three level hierarchical cluster randomized trials. Biometrics 64, 1256-1262.

Högström, G., Nordström, A., Nordström, P., 2015. Aerobic fitness in late adolescence and the risk of early death: a prospective cohort study of 1.3 million Swedish men. Int. J. Epidemiol. 45 (4), 1159-1168. https://doi.org/10.1093/ije/dyv321.

Kelly, N.R., Mazzeo, S.E., Evans, R.K., et al., 2011. Physical activity, fitness and psychosocial functioning of obese adolescents. Ment. Health Phys. Act. 4 (1), 31-37. https://doi.org/10.1016/j.mhpa.2010.11.001.

Kessler, R.C., Amminger, G.P., Aguilar-Gaxiola, S., et al., 2007. Age of onset of mental disorders: a review of recent literature. Curr. Opin. Psychiatry 20 (4), 359.

Kjær, M., 1992. Regulation of hormonal and metabolic responses during exercise in humans. Exerc. Sport Sci. Rev. 20 (1), 161-184.

Lang, J.J., Belanger, K., Poitras, V., et al., 2018a. Systematic review of the relationship between $20 \mathrm{~m}$ shuttle run performance and health indicators among children and youth. J. Sci. Med. Sport 21 (4), 383-397.

Lang, J.J., Tomkinson, G.R., Janssen, I., et al., 2018b. Making a case for cardiorespiratory fitness surveillance among children and youth. Exerc. Sport Sci. Rev. 46 (2), 66-75.

LaVigne, T., Hoza, B., Smith, A.L., et al., 2016. Associations between physical fitness and children's psychological well-being. J. Clin. Sport Psychol. 10 (1), 32-47.

Leahy, A., Eather, N., Smith, J.J., et al., 2019. A school-based physical activity intervention for older adolescents: rationale and study protocol for the Burn 2 Learn cluster randomised controlled trial. Br. Med. J. Open 9, e026029-e29.

Leahy, A.A., Eather, N., Smith, J.J., et al., 2019b. Feasibility and preliminary efficacy of a teacher-facilitated high-intensity interval training intervention for older adolescents. Pediatr. Exerc. Sci. 31 (1), 107-117.

Lubans, D.R., Cliff, D.P., 2011. Muscular fitness, body composition and physical selfperception in adolescents. J. Sci. Med. Sport 14, 216-221.

Lubans, D.R., Morgan, P.J., Callister, R., et al., 2011. Test-retest reliability of a battery of field-based health-related fitness measures for adolescents. J. Sports Sci. 29 (7), 685-693.

Lubans, D.R., Richards, J., Hillman, C.H., et al., 2016a. Physical activity for cognitive and mental health in youth: a systematic review of mechanisms. Pediatrics 138 (3), e20161642. https://doi.org/10.1542/peds.2016-1642.

Lubans, D.R., Smith, J.J., Morgan, P.J., et al., 2016b. Mediators of psychological wellbeing in adolescent boys. J. Adolesc. Health 58 (1), 230-236. https://doi.org/10. 1016/j.jadohealth.2015.10.010.

Morales, P.F., Sánchez-López, M., Moya-Martínez, P., et al., 2013. Health-related quality of life, obesity, and fitness in schoolchildren: the Cuenca study. Qual. Life Res. 22 (7), 1515-1523.

Mücke, M., Ludyga, S., Colledge, F., et al., 2018. Influence of regular physical activity and fitness on stress reactivity as measured with the trier social stress test protocol: a systematic review. Sports Med. 48 (11), 2607-2622.

Muthén, L.K., Muthén, B.O., 1998-2017. Mplus user's guide. Muthén \& Muthén, Los Angeles.

Ortega, F.B., Silventoinen, K., Tynelius, P., et al., 2012. Muscular strength in male adolescents and premature death: cohort study of one million participants. Br. Med. J. 345 (e7279). https://doi.org/10.1136/bmj.e7279.

Ransdell, L.B., Detling, N.J., Taylor, A., et al., 2004. Effects of home- and university-based programs on physical self-perception in mothers and daughters. Women Health 39 (2), 63-81. https://doi.org/10.1300/J013v39n02_05.

Richards, J., Foster, C., Townsend, N., et al., 2014. Physical fitness and mental health impact of a sport-for-development intervention in a post-conflict setting: randomised controlled trial nested within an observational study of adolescents in Gulu, Uganda. BMC Public Health 14 (1), 619. https://doi.org/10.1186/1471-2458-14-619.

Rieck, T., Jackson, A., Martin, S.B., et al., 2013. Health-related fitness, body mass index, and risk of depression among adolescents. Med. Sci. Sports Exerc. 45 (6), 1083-1088. https://doi.org/10.1249/MSS.0b013e3182831db1.

Riiser, K., Ommundsen, Y., Småstuen, M.C., et al., 2014. The relationship between fitness and health-related quality of life and the mediating role of self-determined motivation in overweight adolescents. Scand. J. Public Health 42 (8), 766-772.

Rodriguez-Ayllon, M., Cadenas-Sanchez, C., Esteban-Cornejo, I., et al., 2018. Physical fitness and psychological health in overweight/obese children: a cross-sectional study from the ActiveBrains project. J. Sci. Med. Sport 21 (2), 179-184.

Rudolph, K.D., 2002. Gender differences in emotional responses to interpersonal stress during adolescence. J. Adolesc. Health 4 (Suppl), 3-13.

Ruggero, C.J., Petrie, T., Sheinbein, S., et al., 2015. Cardiorespiratory fitness may help in protecting against depression among middle school adolescents. J. Adolesc. Health 57 (1), 60-65.

Ruiz, J.R., Castro-Piñero, J., España-Romero, V., et al., 2011. Field-based fitness assessment in young people: the ALPHA health-related fitness test battery for children and adolescents. Br. J. Sports Med. 45 (6), 518-524. https://doi.org/10.1136/bjsm.2010. 075341.

Smith, J.J., Eather, N., Morgan, P.J., et al., 2014. The health benefits of muscular fitness for children and adolescents: a systematic review and meta-analysis. Sports Med. 44 (9), 1209-1223. https://doi.org/10.1007/s40279-014-0196-4.

Sweeting, H., West, P., Young, R., et al., 2010. Can we explain increases in young people's psychological distress over time? Soc. Sci. Med. 71 (10), 1819-1830.

Whiteford, H.A., Degenhardt, L., Rehm, J., et al., 2013. Global burden of disease attributable to mental and substance use disorders: findings from the Global Burden of Disease Study 2010. Lancet 382 (9904), 1575-1586.

World Health Organisation, 2005. Promoting Mental Health: Concepts, Emerging Evidence, Practice: A Report of the World Health Organization, Department of Mental Health and Substance Abuse in Collaboration With the Victorian Health Promotion Foundation and the University of Melbourne. World Health Organization, Geneva.

Yeatts, P.E., Martin, S.B., Petrie, T.A., 2017. Physical fitness as a moderator of neuroticism and depression in adolescent boys and girls. Personal. Individ. Differ. 114, 30-35. 